\title{
Pivotal Role of Dosimetry in High Activity 131i Mibg Therapy in Patients Affected by High Risk Relapsed Refractory Neuroblastoma: Towards a Deeper Metastatic Response Understanding
}

Bartolomeo Cassano ( $\sim$ bartolomeo.cassano@gmail.com )

Bambino Gesu Pediatric Hospital: Ospedale Pediatrico Bambino Gesu https://orcid.org/0000-0001-

5747-8230

Milena Pizzoferro

Bambino Gesu Pediatric Hospital: Ospedale Pediatrico Bambino Gesu

Silvio Valeri

Bambino Gesu Pediatric Hospital: Ospedale Pediatrico Bambino Gesu

Claudia Polito

Bambino Gesu Pediatric Hospital: Ospedale Pediatrico Bambino Gesu

Salvatore Donatiello

Bambino Gesu Pediatric Hospital: Ospedale Pediatrico Bambino Gesu

Claudio Altini

Bambino Gesu Pediatric Hospital: Ospedale Pediatrico Bambino Gesu

Maria Felicia Villani

Bambino Gesu Pediatric Hospital: Ospedale Pediatrico Bambino Gesu

\section{Aurora Castellano}

Bambino Gesu Pediatric Hospital: Ospedale Pediatrico Bambino Gesu

\section{Annalisa Serra}

Bambino Gesu Pediatric Hospital: Ospedale Pediatrico Bambino Gesu

Franco Locatelli

Bambino Gesu Pediatric Hospital: Ospedale Pediatrico Bambino Gesu

Maria Carmen Garganese

Bambino Gesu Pediatric Hospital: Ospedale Pediatrico Bambino Gesu

Vittorio Cannatà

Bambino Gesu Pediatric Hospital: Ospedale Pediatrico Bambino Gesu

Original research 
Keywords: High Risk Neuroblastoma, 131I-mIBG High Activity Therapy, Dosimetry, Dose-Response Correlation

Posted Date: January 28th, 2021

DOI: https://doi.org/10.21203/rs.3.rs-152771/v1

License: (1) This work is licensed under a Creative Commons Attribution 4.0 International License. Read Full License 


\title{
Title Page
}

Order and Names of the Authors:

Bartolomeo Cassano ${ }^{1}$,

Milena Pizzoferro²,

Silvio Valeri ${ }^{1}$

Claudia Polito ${ }^{1}$,

Salvatore Donatiello ${ }^{1}$,

Claudio Altini ${ }^{2}$,

Maria Felicia Villani²,

Annalisa Serra ${ }^{3}$,

Aurora Castellano ${ }^{3}$,

Franco Locatelli ${ }^{3,4}$,

Maria Carmen Garganese ${ }^{2}$,

Vittorio Cannatà ${ }^{1}$

Concise Title:

PIVOTAL ROLE OF DOSIMETRY IN HIGH ACTIVITY ${ }^{131}$ I MIBG THERAPY IN PATIENTS AFFECTED BY HIGH RISK RELAPSED REFRACTORY NEUROBLASTOMA: TOWARDS A DEEPER METASTATIC RESPONSE UNDERSTANDING

\author{
Affiliations of the authors: \\ ${ }^{1}$ IRCCS Bambino Gesù Children's Hospital, Medical Physics Unit, Rome, ITALY, \\ ${ }^{2}$ IRCCS Bambino Gesù Children's Hospital, Nuclear Medicine Unit/Imaging Department, Rome, ITALY, \\ ${ }^{3}$ IRCCS Ospedale Pediatrico Bambino Gesu, Department of Pediatric Hematology and Oncology \\ ${ }^{4}$ University La Sapienza, Rome, Italy.
}

e-mail of the corresponding authors:

bartolomeo.cassano@gmail.com

\begin{abstract}
Background: Dosimetry in molecular radiotherapy for personalized treatment is assuming a central role in clinical management of aggressive/relapsed tumors. High-Risk relapsed/refractory metastatic Neuroblastoma (HRrrm-NBL) has a poor prognosis and high-activity ${ }^{131} \mathrm{I}-\mathrm{mIBG}$ therapy could represent a promising strategy.

The aim of this study was to report the absorbed dose to whole-body $\left(\mathrm{D}_{\mathrm{WB}}\right)$, red marrow $\left(\mathrm{D}_{\mathrm{RM}}\right)$ and lesions $\left(\mathrm{D}_{\mathrm{Lesion}}\right)$ correlated to clinical outcome.

Methods: 14 patients affected by HRrrm-NBL were treated with high-activity ${ }^{131}$ I-mIBG therapy (two administrations separated by 15 days). The first administration was weight-based whereas the second one was dosimetry-based (achieving 4 Gy to whole-body). In all patients $D_{W B}$ and $D_{R M}$ has been assessed; 9/14 patients were selected for $D_{\text {Lesion }}$ evaluation (for a total of 13 lesions). Treatment response was classified in Progression and Stable Disease (PD and SD), Partial and Complete Response (PR and CR) and correlated to the value of $\mathrm{D}_{\text {Lesion }}$ value.

Results: The cumulative $\mathrm{D}_{\mathrm{WB}}, \mathrm{D}_{\mathrm{RM}}$ and $\mathrm{D}_{\text {Lesion }}$ ranged from $[1.49 ; 4.45],[0.99 ; 2.56]$ and $[44.17 ; 585.75]$ Gy. After treatment 3, 2, 4 and 5 patients showed CR, PR, SD and PD respectively showing a correlation between the clinical outcome and $\mathrm{D}_{\text {Lesion }}$ with a threshold at $80 \mathrm{~Gy}$.

Conclusions: Our experience shows feasibility of high activity therapy of ${ }^{131}$ I MIBG in rrmHR-NBL children as two administration intensive strategy. Dosimetric approach allowed a tailored high dose treatment maximizing the benefits of radionuclide therapy for pediatric patients with a safety profile. The assesment of $\mathrm{D}_{\text {Lesion }}$ contributed to have a deeper understaning of metabolic treatment effects.
\end{abstract}

Keywords

High Risk Neuroblastoma, ${ }^{131}$ I-mIBG High Activity Therapy, Dosimetry, Dose-Response Correlation 


\section{Declarations}

Ethics approval and consent to participate: All procedures performed in studies involving human participants were in accordance with the ethical standards of the institutional and/or national research committee and with the $1964 \mathrm{Helsinki}$ declaration and its later amendments or comparable ethical standards. Informed consent was obtained from all individual participants included in the study.

Consent for publication: Informed consents for the publication of the study, the diagnostic and therapeutic procedure were obtained from the legally authorized representative.

Availability of data and material: The datasets used and/or analysed during the current study are available from the corresponding author on reasonable request.

Competing interests: All authors declare that they have no conflict of interest.

Funding: No funding to declare

Authors' contributions:

- $\quad$ BC, MP, SV and VC conceived the present studio and wrote the manuscript with support from CP, SD, CA, MFV.

- $\quad \mathrm{BC}, \mathrm{SV}$ and $\mathrm{CP}$ performed the analytic calculations.

- $\quad \mathrm{AS}, \mathrm{AC}$ and FL enroelled all patients

- $\mathrm{MCG}, \mathrm{MP}, \mathrm{CA}$ and MFV collected and analized all clinical informations of the patients.

- All authors read and approved the final manuscript

Acknowledgements: Not Applicable 


\section{Text}

Treatment of relapsed/refractory metastatic high-risk neuroblastoma (rrmHR-NBL) after multimodality therapy remains a clinical challenge; despite recent advances in our understanding of neuroblastoma biology and new treatment strategies, the outcome for children affected by rrmHR-NBL remains poor with an overall survival around 50\% [1,2].

Approximately $90 \%$ of NBL cases accumulate the metaiodobenzylguanidine (mIBG) by a specific uptake mechanism and ${ }^{131} \mathrm{I}$ mIBG can been used for targeted radiotherapy delivering a focal dose of radiation to the tumor sites. A 30-40\% response rate has been observed in refractory and relapsed Neuroblastoma [3,4] and the application of target radiotherapy in new sequential treatments with various drugs is under clinical assessment. Among sequential treatment strategies for rrmHR-NBL, High Dose mIBG can be combined with chemotherapy followed by autologous stem cell transplantation (ASCT) [5, 6]. Thus, children with RRHR-NBL continue to need combinations of different therapeutic modalities to prolong survival and minimize the toxicities of additional therapy.

In 2005, Gaze et al. [7] demonstrated the feasibility of high activity mIBG therapy based on dosimetric approach combined with chemotherapy. The rationale of this study protocol is based on two high activity ${ }^{131} \mathrm{I}-\mathrm{mIBG}$ administrations guided by a dosimetric assessment to achieve a whole-body (WB) absorbed dose of 4 Gy.

This paper will focus on reporting our experience in dosimetric assessment in children affected by rrmHR-NBL treated with high activity MIBG therapy, underlying the correlation between WB and red marrow (RM) absorbed dose and demonstrating the usefulness of lesions dosimetry.

2

Methods

\section{$2.1 \quad$ Patients}

In the present study, 14 patients ( 7 boys and 7 girls with ages ranging from 3 to 17 years) with rrHR-NBL previously treated have been enrolled and submitted to high activity ${ }^{131}$ I-mIBG administrations from 2016 to 2019 at "Bambino Gesù Children Hospital". The age at the time of the initial diagnosis ranged from 1 to 17 years (median 4 years). The treatment protocol was based on a scheme consisting of two ${ }^{131} \mathrm{I}-\mathrm{mIBG}$ administrations 2 weeks apart followed by a single dose of Melphalan $\left(110 \mathrm{mg} / \mathrm{m}^{2}\right) 96$ hours after the second ${ }^{131} \mathrm{I}-\mathrm{MIBG}$ administration and autologous stem cell transplantation 24 hours after the end of chemiotherapy.

First ${ }^{131} \mathrm{I}-\mathrm{mIBG}$ administration was weight-based (about $444 \mathrm{MBq} / \mathrm{kg}$, excepted for 5 patients over $50 \mathrm{~kg}$ of weight who received no more than $17 \mathrm{GBq}$ due to legal limit), whereas the second administration was dosimetry based in order to achieve an absorbed dose to WB ( $\left.\mathrm{D}_{\mathrm{WB}}\right)$ equals to 4 Gy as suggested by Gaze et al. [7].

Thyroid protection block was performed in all patients by administration of Lugol solution and triiodothyronine since 72 $\mathrm{h}$ before the first MIBG treatment to 15 days after the second one. mIBG avid disease was proven by ${ }^{123} \mathrm{I}-\mathrm{mIBG}$ scan to evaluate basal status disease and clinical response after treatment. ${ }^{123} \mathrm{I}-\mathrm{mIBG}$ scans were assessed according SIOPENmIBG score system [8] and the clinical response after ${ }^{131} \mathrm{I}-\mathrm{mIBG}$ therapy was classified in Progression and Stable Disease (PD and SD), Partial and Complete Response (PR and CR) according to the International Neuroblastoma Response Criteria (INRC) Definition of Response [9] within a range from 1 to 3 months.

\subsection{WB/RM Dosimetry}

Each patient was submitted to 6 or 7 dose rate measurements (R) to assess whole-body cumulated activity (Ã $\tilde{w B}_{\text {) }}$ Measurements were performed in anterior and posterior views, using Geiger counter placed at 2 meters of distance from the patient with a duration of 3 minutes. The first measurement $\mathrm{R}(0)$ was performed immediately after radiotracer 
administration, avoiding bladder emptying in order to measure the dose rate of all the administered activity; the next measurements were performed after bladder emptying with the following time sampling $[1 ; 6 ; 24 ; 48 ; 54 ; 166]$ hours after tracer administration; background activity was registered before each time point. The activity at time $t$ was calculated using the following formula:

$$
A_{W B}(t)=A_{A d m} \cdot \sqrt{\frac{R_{A}(t) \cdot R_{P}(t)}{R_{A}(0) \cdot R_{P}(0)}}
$$

where $R_{A}$ e $R_{P}$ are dose rates, in anterior and posterior views, considering background correction.

With an anaolugous time sampling six blood samples have been collected (with a volume of about $3 \mathrm{ml}$ ) using a central line catheter previously placed for clinical purpose. Blood sample activities were to assess after two weeks to reduce dead-time effects, using a gamma counter. Blood activity concentration ([ $\left.\left.\mathrm{A}_{\mathrm{Blood}}\right]\right)$ was calculated as follows:

$$
\left[A_{\text {Blood }}(t)\right]=C P S \cdot \frac{C_{G C}}{m_{\text {blood }}}
$$

Where CPS (counts per second) is the output signal of the gamma counter after a measurement of 10 minutes, $\mathrm{C}_{\mathrm{GC}}$ is the gamma counter calibration factor which transforms CPS into activity and $m_{\text {Blood }}$ is the mass of the blood of each sample. The calculation of $\tilde{\mathrm{A}}_{\mathrm{WB}}$ and [ $\left.\tilde{\mathrm{A}}_{\mathrm{Blood}}\right]$ has been performed using by NukFit software [10]. With this software it was possible to choose the best fit model, competing a mono and bi exponential fit using the AICc method [11], and to calculate the area under the curve.

According to EANM guidelines $\mathrm{D}_{\mathrm{WB}}$ has been calculated using the following formulas [12]:

$$
D_{W B}=\tilde{A}_{W B} \cdot S_{W B \leftarrow W B}
$$

Using an S-factors. given by the following formula [13, 14]:

$$
S_{W B \leftarrow W B}=1.3410^{-4} m_{p}^{-0.921}\left[\frac{G y}{M B q \cdot h}\right]
$$

where $m_{p}$ is the patient's weight.

Whereas, The $\mathrm{D}_{\mathrm{RM}}$ has been calculated using the formula showed in $[15,16]$ :

$$
\begin{gathered}
D_{R M}=R M B L R\left[\tilde{A}_{\text {blood }}\right] m_{R M} S_{R M \leftarrow R M}+ \\
+\left(\tilde{A}_{W B}-R M B L R\left[\left[\tilde{A}_{\text {Blood }}\right] m_{R M} \frac{m_{p}}{m_{W B}}\right) \cdot \frac{m_{T B}}{m_{p}} \cdot S_{R M \leftarrow R o B}\right.
\end{gathered}
$$

Where $m_{W B}$ and $m_{R M}$ are body mass and RM mass of standard phantom, respectively; RMBLR is instead radioactive concentration ratio between marrow and blood, set equal to 1 and S-Factors are taken by Olinda [17].

\subsection{Lesion Dosimetry}

The conjugate views method described in Pamhlet MIRD 16 [18] was used to calculate the absorbed dose by patient's lesions using static planar scintigraphic images. Evaluation of 13 lesions in 9 patients detailed the following features: a) lesions were qualitatively detectable with tumor delineation feasible both on CT images and on Scintigrafic images after the administration of ${ }^{131} \mathrm{I}-\mathrm{mIBG}$; b) lesions were to be non-retrohepatic.

Each of this patient underwent on the following protocol of acquisition consisting of a CT scan, and a trasmissive acquisition (before the first administration of ${ }^{131} \mathrm{I}-\mathrm{mIBG}$ ) and a series of static images after the first and the second therapeutic administration.

A CT scan of the body's segment including the lesion's site was performed within a range of 1-3 months before radioiodine treatment. By contouring the tumor's edges on this scan, the volume and the mass of the lesion were calculated considering a density equal to 1 in case of soft tissue. 
In order to estimate the patient's body thickness along lesion's view (T), one hour before radiotracer administration, two transmissive images (one without the patient -blank- and the other one with the patient) were acquired using a source of ${ }^{99 \mathrm{~m}} \mathrm{Tc}$ - of about $37 \mathrm{MBq}$ and LEHR collimators.

A series of static scintigraphic acquisition has been collected after the first and the second administration. The acquisition parameters were reported in table 1.

The scintigraphic images were subsequently corrected for scattering, using the Ogawa method [19], and for dead time placing a known source (of about 11.1 MBq) in the field of view, avoiding the overlap on patient's body and normalising the total counts acquired within the image to those obtained from the source without the patient present [20]. The lesion was delineated on the first image of the series and, in order to improve the placement of lesion's region of interest (ROI), all (scintigraphic and trasmissive) planar images were co-registered referring to the first scintigraphic image acquired.

Following the Pamphlet MIRD 16 lesion's activity at time t was calculated according to the formula showed in

$$
A_{\text {lesion }}(t)=\sqrt{\frac{I_{\text {Ant }}(t) \cdot I_{\text {Post }}(t)}{e^{-\mu(I-131) \cdot T}}} \cdot \frac{f_{j}}{C}
$$

Where $I_{A n t}$ and $I_{\text {Post }}$ and counts inside lesion's ROI, in anterior and posterior views respectively, corrected for background by Bujiis' method; $\mathrm{f}_{\mathrm{j}}$ is the self attenuation correction; $\mathrm{C}$ and $\mu(I-131)$ are the sensitivity of the gamma camera and the attenuation coefficient for ${ }^{131} \mathrm{I}$ obtained using an abosolute quantification described in [15, 21] and are equal to $12.83 \mathrm{cps} / \mathrm{MBq}$ and $0.106 \mathrm{~cm}^{-1}$ respectively.

Finally the absorbed dose to lesion $\mathrm{D}_{\text {Lesion }}$ was calculated using the formula:

$$
D_{\text {Lesion }}=\tilde{A}_{\text {Lesion }} \cdot S_{\text {Lesion } \leftarrow \text { Lesion }}
$$

Where $\tilde{A}_{\text {Lesion }}$ is the cumulated activity, calculated by Nukfit in the same way as described for the WB and $S_{\text {Lesion } \leftarrow \text { Lesion }}$ using the $\mathrm{S}$ values of the spheres taken from the Olinda software.

3

\section{Results}

\subsection{Clinical Results}

In table 2 the main patient's characteristics and the relative clinical response to ${ }^{131} \mathrm{I}-\mathrm{mIBG}$ therapy are summarized. All Patients had a progressive disease at ${ }^{123}$ I MIBG pre-therapy scan showing a SIOPEN score greater than 3. After ${ }^{131}$ I-mIBG therapy 5 /14 Patients (35.7\%) showed a progressive disease while 4/14 (28.6\%) patients showed stable disease. Two patients $(14.3 \%)$ showed partial response and three (21.4\%) patients showed a complete response.

The "responding group" rate, considering all those patients who had a therapeutic effect (such as complete response, partial response or stable disease), was 64.3\%. No-responding group" rate (progression disease) was 35.7\%.

\subsection{Absorbed dose to WB/RM Results}

On the base of the $\mathrm{D}_{\mathrm{WB}}$ assessment at the first administration, 13 patients received a full treatment while only one patient received a single dose administration due to the achievement of a $\mathrm{D}_{\mathrm{WB}}$ value close to $4 \mathrm{~Gy}$ ( $4.2 \mathrm{~Gy}$ ). The injected activities at the second administration to obtain a complete treatment were in the range of $[1.84 ; 17.90] \mathrm{GBq}$ with a median of 11.00 $\mathrm{GBq}$ while the total activity administered was between 2.2 and $35.4 \mathrm{GBq}$ (median $20.8 \mathrm{GBq}$ ).

The ranges of abosrbed dose values for single administration, the absorbed dose obtained summing the results of the two administrations and the normalized dose to administered activity $\left(\mathrm{D}_{\mathrm{WB}} / \mathrm{A}\right)$ were reported in table 3.

In Fig 1 were reported cumulated doses to WB in 14 treated patients.

Considering the 13 patients who were administered the dose twice, the differential percentage of the $\mathrm{D}_{\mathrm{WB}} / \mathrm{A}$ (normalized dose to administered activity) between the first and second administration, was between $-37.0 \%$ and $18.5 \%$ with a median of $-16 \%$. 8 out of 13 patients $(61.5 \%)$ showed a reduction of the dose per unit of administered activity between the first 
and second treatment while 4 out of 13 patients (30.8\%) showed an increase of $\mathrm{D}_{\mathrm{wB}} / \mathrm{A}$ inferior to $10 \%$. In Fig 2 were reported D/A values of 13 patients who received two administrations and Table 3 shows dosimetric results referring to WB.

The percentage difference of $\mathrm{D}_{\mathrm{RM}} / \mathrm{A}$ between the first and the second administration was included between $-57.0 \%$ and $13.1 \%$ with a median value of $-23.8 \%$. In particular, $9 / 13$ patients $(69.23 \%)$ showed a reduction of D/A, while $3 / 13$ patients $(23.08 \%)$ showed an increase of $\mathrm{D}_{\mathrm{RM}} / \mathrm{A}$ inferior to $10 \%$. In Fig 3were reported $\mathrm{D}_{\mathrm{RM}} / \mathrm{A}$ values of 13 patients who received two administrations and Table 4 shows dosimetric results referring to RM.

In Fig 4 the trend of the dose to RM respect to the dose to WB are reported. The graph shows a strong linear correlation between the two parameters with an angular coefficient and a $\mathrm{R}^{2}$ value equal to 1.7 and 0.9922 respectively.

\subsection{Absorbed dose to lesion Results}

The number of detected lesion following the mentioned inclusion criteria was 13 in 9 patients and their anatomical features and dosimetric results were reported in table 5.

Their distribution in different anatomical districts was heterogeneous with a volume range of $0.40-246.38 \mathrm{~cm}^{3}$ from the cervical district to the low abdomen.

The absorbed dose on a single administration was included between 6.3 and 300.9 Gy with a median 81.8 Gy while the total absorbed dose of the two administrations was in the range [44.2; 585.8] Gy, with a median of 164.3 Gy. The mean of the doses after the two administrations was included between 22.1 and 292.9 Gy with a median of 94.6 Gy; $\mathrm{D}_{\text {Lesion }} / \mathrm{A}$ was within the range of $[0.94 ; 26.10] \mathrm{Gy} / \mathrm{GBq}$ with a median of $8.59 \mathrm{~Gy} / \mathrm{GBq}$. The percentage difference in $\mathrm{D}_{\text {Lesion }} / \mathrm{A}$ between the second and the first administrazion was included between $-76.8 \%$ and $21.0 \%$ with a median of $-44.7 \%$. Fig 5 and table 5 show the dosimetric values of 13 lesions in 9 patients. 10/13 (77\%) lesions show a reduction of the uptake at the second administration compared to the first one (percentage differences range from $-76.9 \%$ to $-22.6 \%$ ); $2 / 13$ $(15.4 \%)$ lesions have a quite similar values and only in one case $(7.7 \%)$ there is an increase of the uptake equals to 21.0 $\%$. In Fig 5 were reported D/A values of 13 lesions for each administration.

We also assessed a possible therapeutic dose-response correlation. The doses to the average lesions were divided into two categories: a)"Responding”, all those patients who had a therapeutic effect such as complete response, partial response or stable disease; b) "No Responding", all those patients who did not show a positive response to treatment (progression disease). Results were reported in Fig 6.

The median of the No-Responding group was 53.1 Gy, while that one of the Responding group was 129.2 Gy. The minimum value present in the responding group was equal to $42.7 \mathrm{~Gy}$. The Mann-Whitley test showed that the median absorbed doses were significantly different in the two groups $(\mathrm{p}=0.0196)$

\section{Discussion}

Our experience shows feasibility of high activity therapy of ${ }^{131}$ I MIBG in rrmHR-NBL children as two administration intensive strategy. As shown by Genolla et al. [22], the use of personalized dosimetry in combination with the clinical data confirms that tandem protocol theorized by Gaze et al [7] is a safety treatment.

Dosimetric approach allows a tailored high dose treatment maximizing the benefits of radionuclide therapy for pediatric patients.

The graph reported in Fig 1 shows that the summed dose value after the two administrations was close to the dose of 4 Gy in 10 patients (in the range of $3-4.5$ Gy). The remaining 4 patients reached a dose to WB within the range [1.5; 2.9] since it was not possible to administer a higher activity than $17 \mathrm{GBq}$ due to legal limits. 
In 2015 Minguez et Al. [23] studied the correlation between $\mathrm{DWB}_{\mathrm{WB}} / \mathrm{A}$ and patient's mass, using data reported in 3 different studies $[23,24,25]$ and obtaining the following relationship:

$$
\frac{D_{W B}}{A}=3.63 m^{-0.921}
$$

The results of this work confirmed this relation obtaining the following similar result:

$$
\frac{D_{W B}}{A}=3.48 m^{-0.921}
$$

From the comparison of the two results (Fig 7) it is possible to note that the two curves are very similar with a percentage difference equals to $4.3 \%$. The correlation is very strong as underlined by the $\mathrm{R}^{2}$ value equals to 0.9214 . However the maximum distance between data and fit is around $43 \%$, consequently, equation number 13 can be used in a first approximation for the choice of the activity to be administered but patient's dosimetry is still necessary in order to obtain a reliable dose value.

Generally the calculation of the absorbed dose to RM is not performed for patient affected by rrmHR-NBL treated with two administration of ${ }^{131} \mathrm{I}-\mathrm{mIBG}$. Blood sample collection is not considered as an invasive method due to the presence of a CLC generally placed for clinical purpose in oncologist patient submitted to heavy treatment. For this reason it has been possible to collect 7 blood samples from each patient. The quantitative analysis of the $D_{R M}$ allowed evaluating the dosimetry respect to the clinical response. In the present protocol, the dose limit to WB of the entire treatment is set at 4 Gy without considering quantitative prescriptions on dosimetry to RM. Reviewing the literature about analogous dosimetric protocol, Gaze et al. sets the dose limit to red marrow at 2Gy [26] in order to avoid marrow toxicity. Analyzing the $\mathrm{D}_{\mathrm{RM}}$, the DRM values were higher than $2 \mathrm{~Gy}$ in $50 \%$ of patients (maximum value $2.56 \mathrm{~Gy}$ ). This result is not in contradiction with the literature data because myeloablation is a deterministic effect required in the treatment protocol in preparation for bone marrow transplantation.

Considering the $\mathrm{D}_{\mathrm{wB}} / \mathrm{A}$ values (showed in Fig 2 and Fig 3 for WB and RM respectively) and calculating the percentage difference between the first and the second administration is possible to assume that the first result is predictive of the second one; infact in the majority of the cases (9/13) the differences is lower than $10 \%$.

Fig 4 shows the correlation between $\mathrm{D}_{\mathrm{WB}}$ and $\mathrm{D}_{\mathrm{RM}}$ confirming the linear correlation obtained by Minguez et Al. with a percentage differences between the angular coefficients equals to $6.25 \%$.

It should be considered that dosimetric implementation in pediatric practice requires peculiar skills and a dedicated multidisciplinary team (pediatric nuclear medicine staff, oncologists, medical physicists) in order to obtain the best collaboration according to the patient's age. Furthermore, in literature there is a limitated experience in dosimetric approach about radionuclide therapy in pediatric field; a dosimetric assessment including $\mathrm{D}_{\mathrm{RM}}$ and $\mathrm{D}_{\mathrm{WB}}$ could be helpful to have a complete data panel to define a reliable standardized procedure.

In order to have a deeper understanding about lesion uptake and relative metastatic response, we assessed absorbed dose to lesions when feasible by planar dosimetric approach.

Concerning the lesions dosimetry, the doses distribution shows a wide range of calculated values on a single administration $[6.2 ; 300.9]$ Gy. This variability depends on the lesion's volume which is in the range $[0.4 ; 246.4] \mathrm{cm}^{3}$. Furthermore, the obtained results are comparable with those reported in literature in similar studies regarding to the absorbed dose to lesions [27].

Analizing Fig 5 and table 5, a high number of lesions appears to have an uptake reduction between the first and second administration, due to radiosensitivity phenomenon and to the reduction of the lesion size. Therefore it is possible to state that the first administration may not be predictive for the second which will take place with a lower dose. 
Fig 8 summarizes all clinical efforts to overcome the complex aspects linked to this challenge disease reporting a case of complete response. Otherwise, analysing "no responding" cases by dosimetric data allows to a have a deeper understanding of limitative factors to obtain a therapeutic response.

In Fig 6 it is possible to note that in our cases there is a distinction between the two groups of response (Responding and no Responding) with a threshold value near to $80 \mathrm{~Gy}$. This obtained value is the same reported in [28] regarding to the absorbed dose to lesion in thyroid cancer. By further investigations and a high number of cases, it's crucial to understand if absorbed dose to lesion could give important prognostic information predicting Patient's treatment response.

The main drawbacks of the present study are due to limits of planar method which doesn't allow dosimetric assessment for overlapped uptake areas. In most of our cases, lesions were placed in retrohepatic site and the physiological liver uptake limited the applicability of planar dosimetric approach. SPECT/CT imaging for tumor dosimetry (3D and voxel dosimetry) overcomes planar limits about critical site and lesion heterogeneity, expecially in neuroblastoma disease.

Recent trials have focused on integrated treatment strategy in order to improve current poor prognosis in rrmHR-NBL patients. VERITAS trial [29] represents a promising therapeutic approach for neuroblastoma clinical management applying intensive high-dose radioiodine treatment protocol in an early stage of disease in case of poor response to induction chemotherapy. Furthermore, multicetric approach of VERITAS trial allows to obtain stronger results overcoming the limited number of patients with this rare disease submitted to high doses radioiodine treatment. Tailored treatment approaches based on dosimetry allows to include radionuclide therapy into systemic chemotherapy and myeloablative transplantation protocols maximizing the treatment benefits ensuring a high safety profile. Our experience enforces the confidence the personalized dosimetry could give a significant contribution in patient care affected by highrisk neuroblastoma.

\section{5}

Compliance with Ethical Standards

All authors declare that they have no conflict of interest.

All procedures performed in studies involving human participants were in accordance with the ethical standards of the institutional and/or national research committee and with the 1964 Helsinki declaration and its later amendments or comparable ethical standards.

Informed consent was obtained from all individual participants or their legal guardian included in the study

The present protocol study has been approved by our local ethics committee

\section{References}

1. NR Pinto, MA Applebaum, SL Volchenboum et al. Advances in Risk Classification and Treatment Strategies for Neuroblastoma. J Clin Oncol. 2015 Sep 20;33(27):3008-17. doi: 10.1200/JCO.2014.59.4648

2. DA Morgenstern, G Barone, L Moreno et al. Options for the treatment of patients with relapsed/progressive high-risk neuroblastoma. Children's Cancer and Leukaemia Group, Neuroblastoma Special Interest Group. March 2015.

https://www.cclg.org.uk/write/MediaUploads/Member\%20area/Treatment\%20guidelines/CCLG_Relapsed_Prog ressive_High_Risk_Neuroblastoma_Guidelines_March_2015_FINAL.pdf

3. KK Matthay, G Yanik, J Messina et al. Phase II study on the effect of disease sites, age, and prior therapy on response to iodine-131-metaiodobenzylguanidine therapy in refractory neuroblastoma. J Clin Oncol. 2007 Mar 20;25(9):1054-60. doi: 10.1200/JCO.2006.09.3484

4. JS Wilson, JE Gains, V Moroz et al. A systematic review of 131I-meta iodobenzylguanidine molecular radiotherapy for neuroblastoma. Eur J Cancer. 2014 Mar;50(4):801-15. doi: 10.1016/j.ejca.2013.11.016 
5. KK Matthay, JC Tan, JG Villablanca et al. Phase I dose escalation of iodine-131-metaiodobenzylguanidine with myeloablative chemotherapy and autologous stem-cell transplantation in refractory neuroblastoma: a new approaches to Neuroblastoma Therapy Consortium Study. J Clin Oncol. 2006 Jan 20;24(3):500-6. doi: 10.1200/JCO.2005.03.6400

6. S French, SG DuBois, B Horn et al. 131I-MIBG followed by consolidation with busulfan, melphalan and autologous stem cell transplantation for refractory neuroblastoma. Pediatr Blood Cancer. 2013 May;60(5):87984. doi: $10.1002 / \mathrm{pbc} .24351$

7. MN Gaze, YC Chang, GD Flux et al. Feasibility of dosimetry-based high-dose 131I-meta-iodobenzylguanidine with topotecan as a radiosensitizer in children with metastatic neuroblastoma. Cancer Biother Radiopharm. 2005 Apr;20(2):195-9. doi: 10.1089/cbr.2005.20.195

8. V Lewington, B Lambert, U Poetschger et al. ${ }^{123}$ I-mIBG scintigraphy in neuroblastoma: development of a SIOPEN semi-quantitative reporting, method by an international panel. Eur J Nucl Med Mol Imaging. 2017 Feb;44(2):234-241. doi: 10.1007/s00259-016-3516-0

9. JR Park, R Bagatell, SL Cohnet al. Revisions to the International Neuroblastoma Response Criteria: A Consensus Statement From the National Cancer Institute Clinical Trials Planning Meeting. J Clin Oncol. 2017 Aug 1;35(22):2580-2587. doi: 10.1200/JCO.2016.72.0177

10. P Kletting, S Schimmel, HA Kestleret al. Molecular radiotherapy: the NUKFIT software for calculating the time-integrated activity coefficient. Med Phys. 2013 Oct;40(10):102504. doi: 10.1118/1.4820367.

11. G Glatting, P Kletting, SN Reske et al. Choosing the optimal fit function: comparison of the Akaike information criterion and the F-test. Med Phys. 2007 Nov;34(11):4285-92. doi: 10.1118/1.2794176

12. C Hindorf, G Glatting, C Chiesa et al. EANM Dosimetry Committee. EANM Dosimetry Committee guidelines for bone marrow and whole-body dosimetry. Eur J Nucl Med Mol Imaging. 2010 Jun;37(6):1238-50. doi: $10.1007 / \mathrm{s} 00259-010-1422-4$

13. SE Buckley, FH Saran ,MN Gaze MN et al. Dosimetry for fractionated (131)I-mIBG therapies in patients with primary resistant high-risk neuroblastoma: preliminary results. Cancer Biother Radiopharm. 2007 Feb;22(1):105-12. doi: 10.1089/cbr.2007.301

14. MG Stabin, JA Siegel. Physical models and dose factors for use in internal dose assessment. Health Phys. 2003 Sep;85(3):294-310. doi: 10.1097/00004032-200309000-00006

15. C Chiesa, L Indovina,C Traino et al. Dosimetria durante terapia del carcinoma differenziato della tiroide metastatico Protocollo Dosimetrico. 2008 https://www.aimn.it/pubblicazioni/LG/protocollo_dosimetrico_meta_cdt.pdf

16. MG Stabin, JA Siegel, RB Sparks. Sensitivity of model-based calculations of red marrow dosimetry to changes in patient-specific parameters. Cancer Biother Radiopharm. 2002 Oct;17(5):535-43. doi: $10.1089 / 108497802760804763$

17. MG Stabin, RB Sparks, E Crowe. OLINDA/EXM: the second-generation personal computer software for internal dose assessment in nuclear medicine. J Nucl Med. 2005 Jun;46(6):1023-7

18. JA Siegel, SR Thomas, JB Stubbs et al. MIRD pamphlet no. 16: Techniques for quantitative radiopharmaceutical biodistribution data acquisition and analysis for use in human radiation dose estimates. J Nucl Med. 1999 Feb;40(2):37S-61S

19. K Ogawa, Y Harata, T Ichihara et al. A practical method for position-dependent Compton-scatter correction in single photon emission CT. IEEE Trans Med Imaging. 1991;10(3):408-12. doi: 10.1109/42.97591 
20. J Gear, C Chiesa, M Lassmann et al. EANM Dosimetry Committee. EANM Dosimetry Committee series on standard operational procedures for internal dosimetry for 131I mIBG treatment of neuroendocrine tumours. EJNMMI Phys. 2020 Mar 6;7(1):15. doi: 10.1186/s40658-020-0282-7

21. G Sgouros. Blood and bone marrow dosimetry in radioiodine therapy of thyroid cancer. J Nucl Med. 2005 May;46(5):899-900

22. J Genolla, $\mathrm{T}$ Rodriguez, $\mathrm{P}$ Minguez et al. Dosimetry-based high-activity therapy with 131Imetaiodobenzylguanidine (131I-mIBG) and topotecan for the treatment of high-risk refractory neuroblastoma. Eur J Nucl Med Mol Imaging. 2019 Jul;46(7):1567-1575. doi: 10.1007/s00259-019-04291-X

23. P Mínguez, G Flux, J Genollá et al. Dosimetric results in treatments of neuroblastoma and neuroendocrine tumors with (131)I-metaiodobenzylguanidine with implications for the activity to administer. Med Phys. 2015 Jul;42(7):3969-78. doi: 10.1118/1.4921807

24. J Toporski, M Garkavij, J Tennvall J et al. High-dose iodine-131-metaiodobenzylguanidine with haploidentical stem cell transplantation and posttransplant immunotherapy in children with relapsed/refractory neuroblastoma. Biol Blood Marrow Transplant. 2009 Sep;15(9):1077-85. doi: 10.1016/j.bbmt.2009.05.007

25. SE Buckley, SJ Chittenden, FH Saran et al. Whole-body dosimetry for individualized treatment planning of 131I-MIBG radionuclide therapy for neuroblastoma. J Nucl Med. 2009 Sep;50(9):1518-24. doi: 10.2967/jnumed.109.064469

26. F Giammarile, A Chiti, M Lassmann et al. EANM. EANM procedure guidelines for 131I-metaiodobenzylguanidine (131I-mIBG) therapy. Eur J Nucl Med Mol Imaging. 2008 May;35(5):1039-47. doi: $10.1007 /$ s00259-008-0715-3

27. KK Matthay, C Panina, J Huberty et al. Correlation of tumor and whole-body dosimetry with tumor response and toxicity in refractory neuroblastoma treated with (131)I-MIBG. J Nucl Med. 2001 Nov;42(11):1713-21

28. HR Maxon, SR Thomas, VS Hertzberg et al. Relation between effective radiation dose and outcome of radioiodine therapy for thyroid cancer. N Engl J Med. 1983 Oct 20;309(16):937-41. doi: 10.1056/NEJM198310203091601

29. Trial Evaluating and Comparing Two Intensification Treatment Strategies for Metastatic Neuroblastoma Patients With a Poor Response to Induction Chemotherapy (VERITAS).

https://clinicaltrials.gov/ct2/show/NCT03165292 
Figures

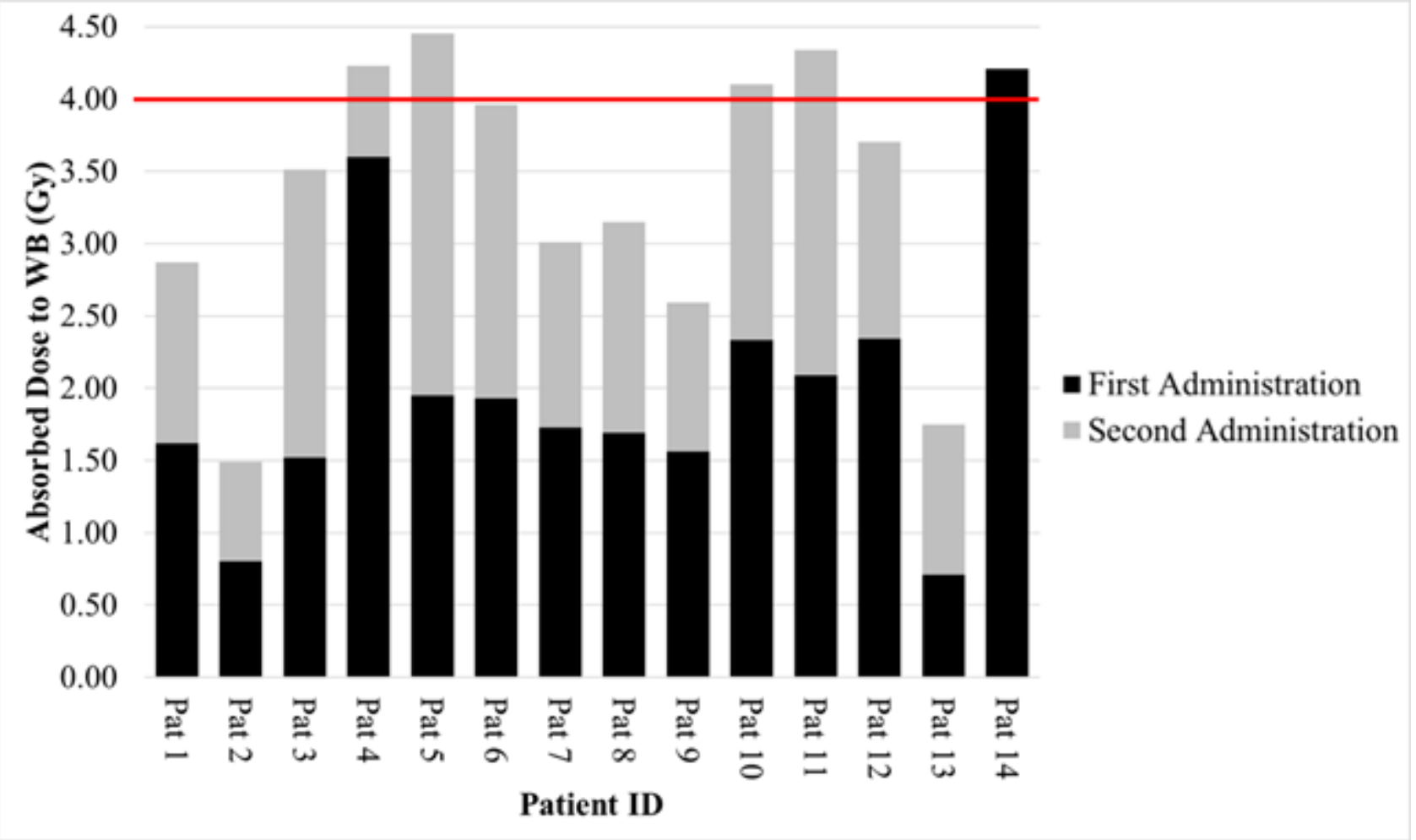

Figure 1

total dose to WB distribution referring to the limit threshold of $4 \mathrm{~Gy}$ (red line), after two treatments (excluding patient number 14 who didn't receive the second treatment).

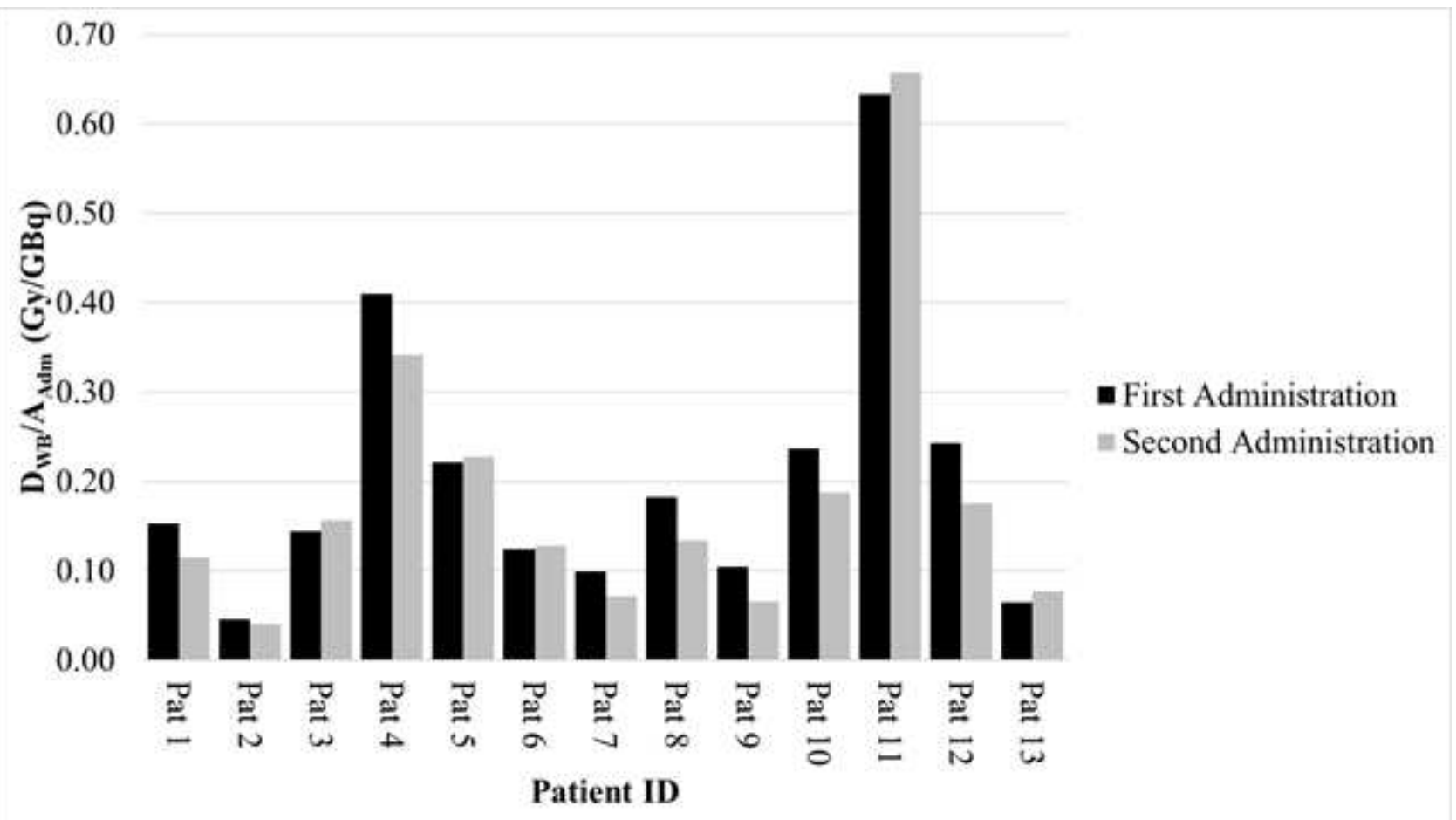

Figure 2 
comparison of dose to WB on administered activity between the first (grey) and second (black) administration.

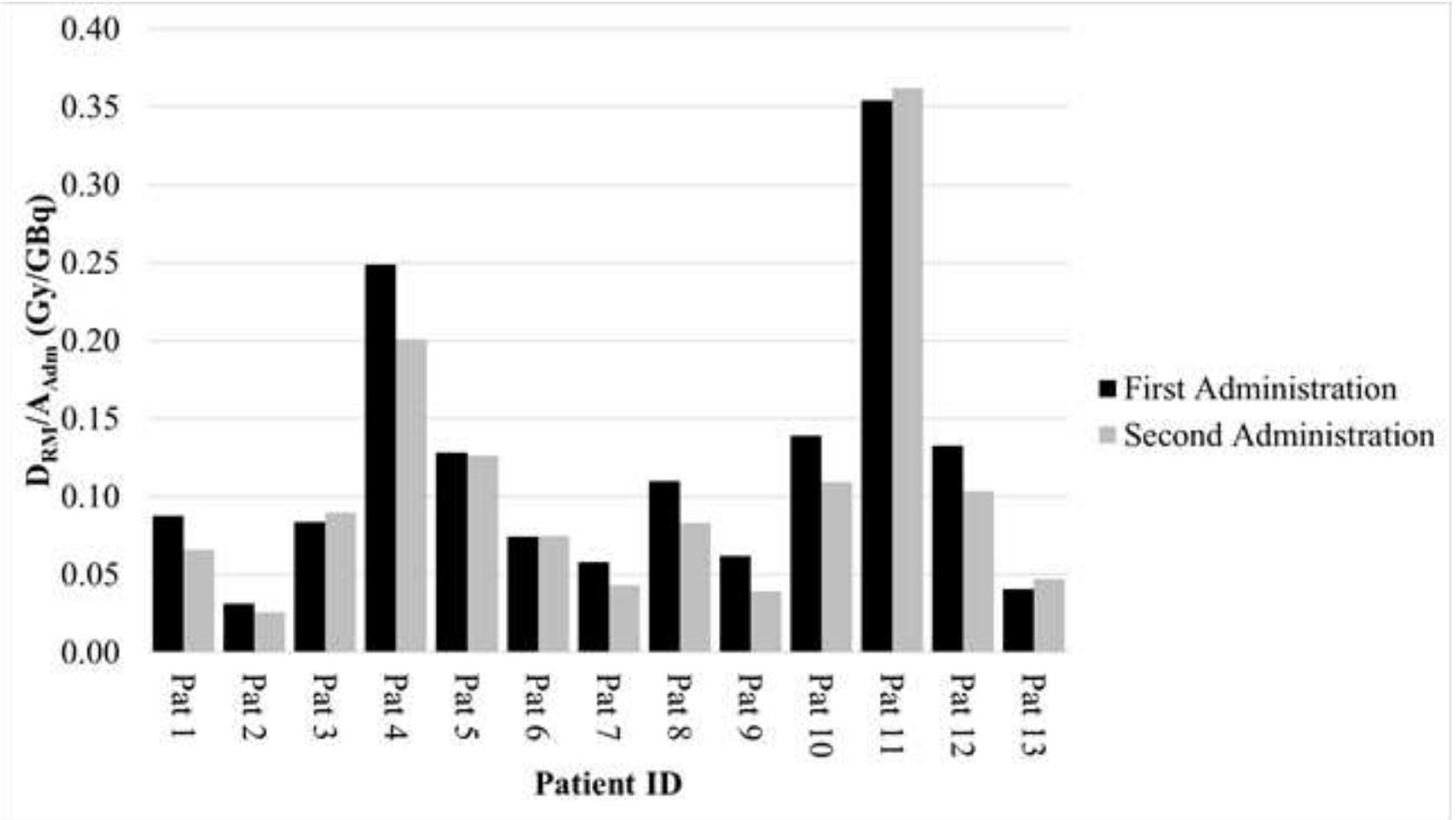

Figure 3

dose comparison of RM on administered activity between the first (grey) and second (black) administration.

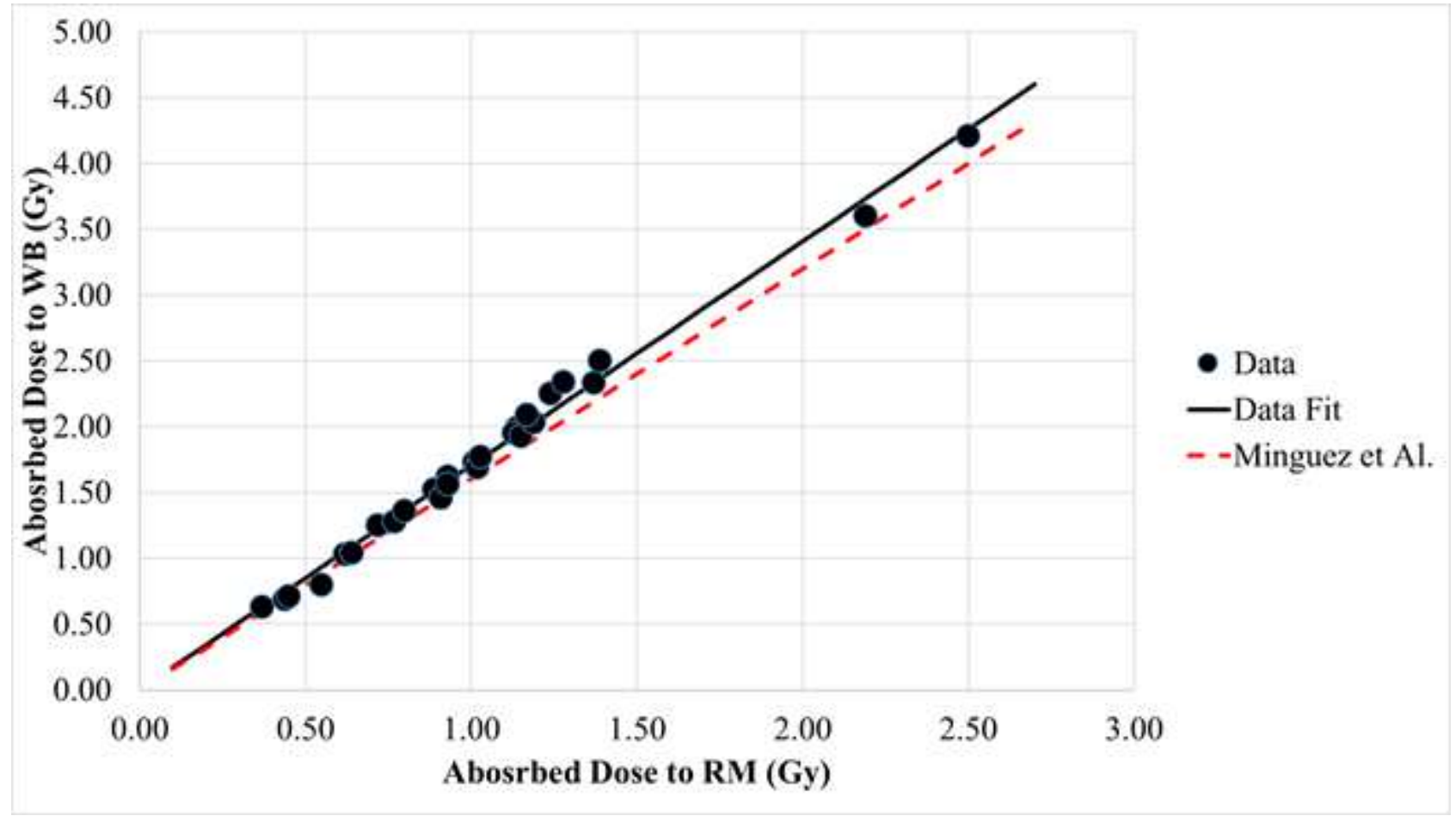

Figure 4 
trend of the dose to RM in respect to the dose to WB and relative fit.

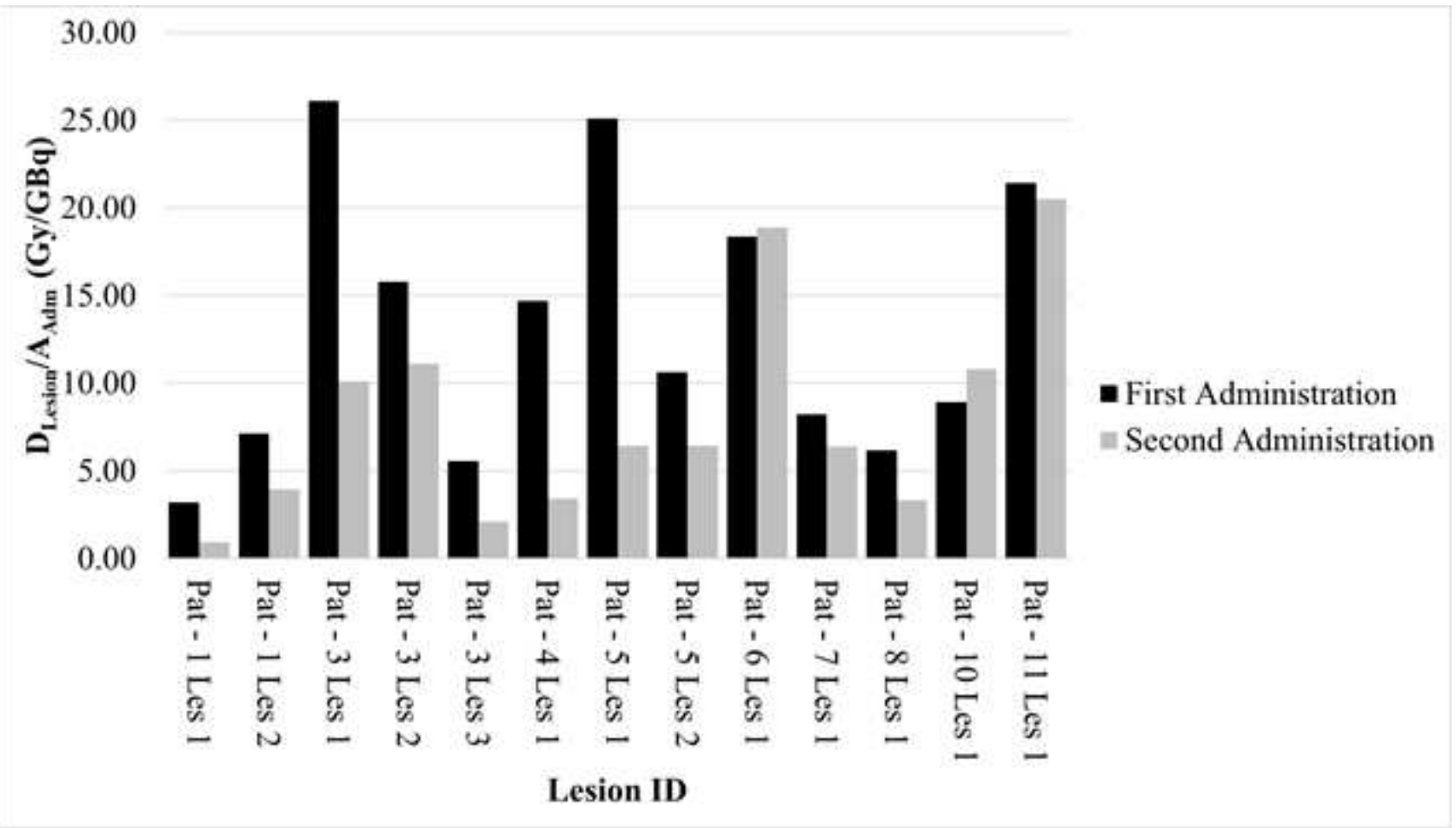

Figure 5

comparison of dose to lesions on administered activity between the first (black) and second (grey) administration.

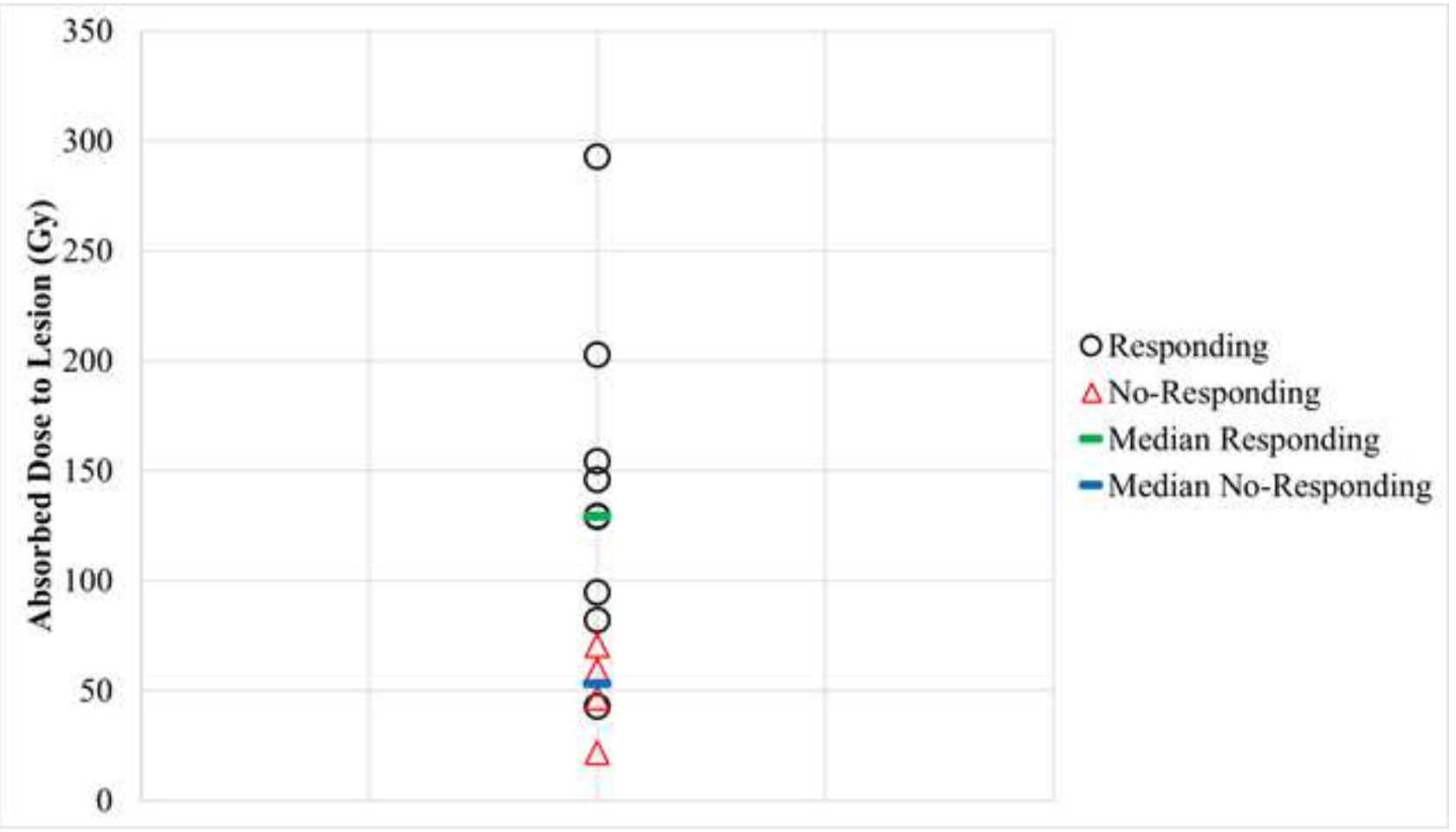

Figure 6 
therapeutic dose-response graph. The average doses to lesions are divided into two groups based on the clinical response. Qualitatively, points of the two groupings are concentrated around different median values.

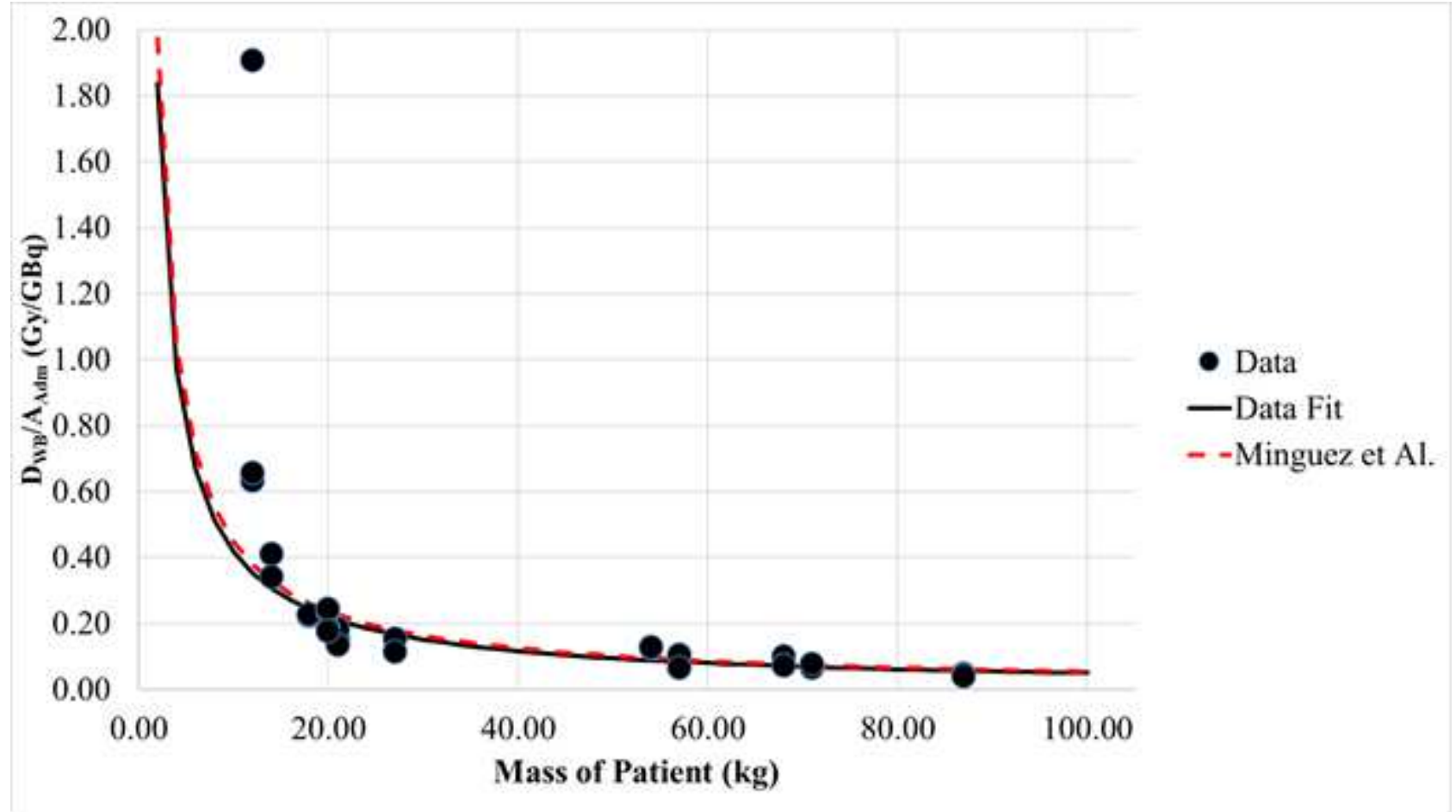

\section{Figure 7}

comparison between Minguez's fit and experimental fit of D/A obtained data versus the mass of the patients.
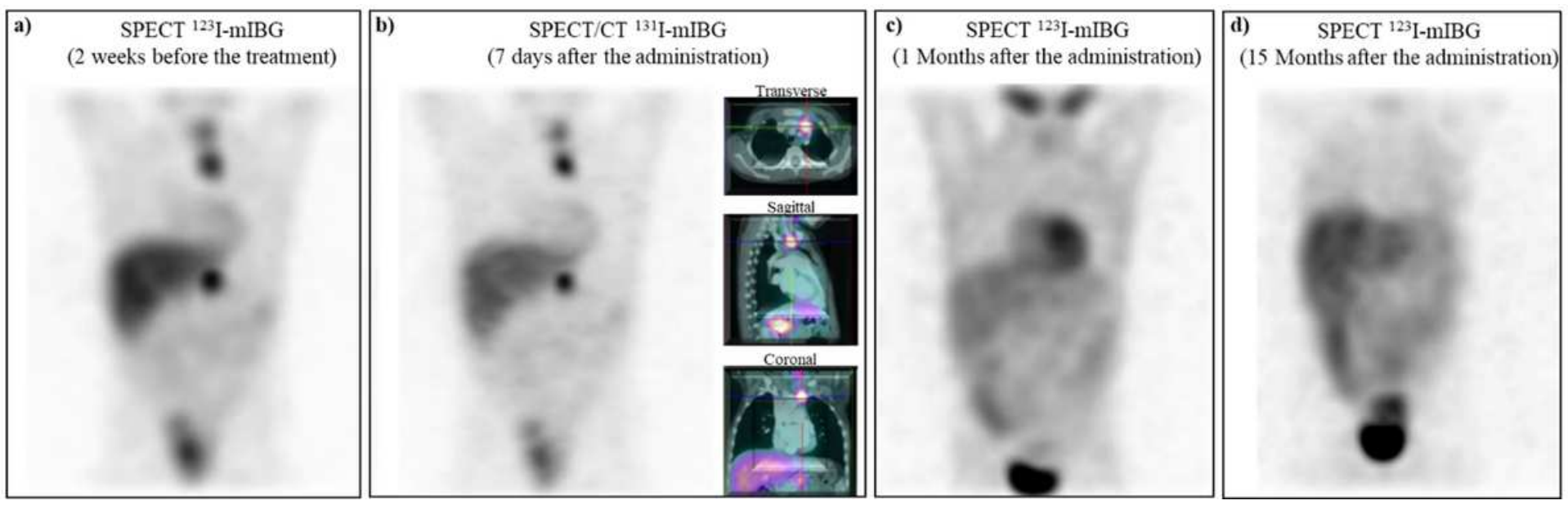

Figure 8

A 4 years old girl with diagnosis of Stage IV NBL at 2 years (abdominal mass associated with cervical and thoracic localization and bone involvement) was treated according to SIOPEN NBHR 01 protocol. At the end of induction chemotherapy, a new balance of disease by mIBG scan showed a complete skeletal response with a partial response of soft tissue lesions. Abdominal residual mass was submitted to partial surgical resection and radiotherapy while cervical and thoracic lesions showed a progression of disease. 
The patient was referred to radioiodine high activity therapy after pre-treatment $123 \mathrm{I} \mathrm{mIBG}$ evalutation(as shown in figure 8a) and confirmed by imaging after radioiodine therapy (Fig8b). The DLesion was equal to $129.23 \mathrm{~Gy}$ after the unique administration. The patient showed an early complete response at 1 month after the 131I-mIBG administration (Fig8c) and confirmed at last followup (Fig. 8d) 